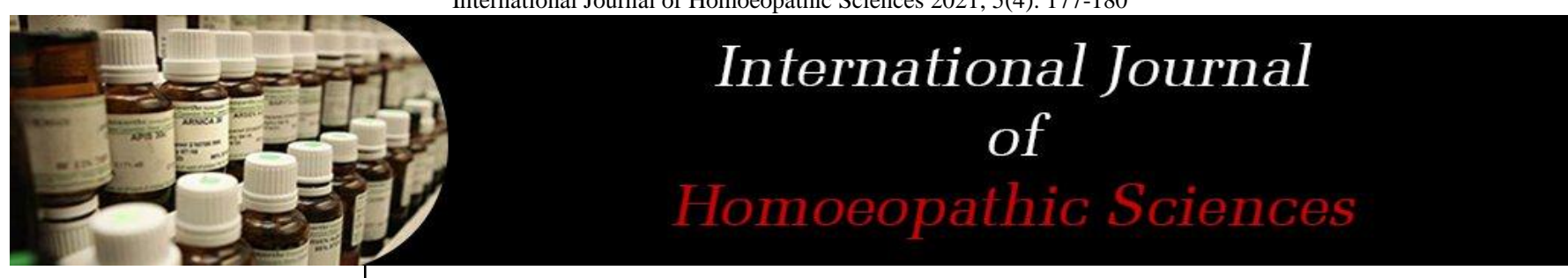

E-ISSN: 2616-4493 P-ISSN: 2616-4485 www.homoeopathicjournal.com IJHS 2021; 5(4): 177-180

Received: 02-08-2021

Accepted: 12-09-2021

Dr. Km Om Jee Assistant Professor, Department Obstetrics and Gynaecology, Dr. Yaduvir Sinha Homoeopathic Medical College and Hospital, Laheriasarai, Darbhanga, Bihar, India
Corresponding Author: Dr. Km Om Jee Assistant Professor, Department Obstetrics and Gynaecology, Dr. Yaduvir Sinha Homoeopathic Medical College and Hospital, Laheriasarai, Darbhanga, Bihar, India

\section{Leucorrhoea and its homoeopathic approach}

\section{Dr. Km Om Jee}

DOI: $\underline{\text { https://doi.org/10.33545/26164485.2021.v5.i4c.469 }}$

\section{Abstract}

Leucorrhoea is one of the major problems encountered in Gynaecological practice. The most common cause of leucorrhoea is physiological, followed by vaginal infections due to bacteria, virus, fungi and parasites. Other causes include foreign bodies, cervicitis and atrophic vaginitis. Homoeopathic medicines for leucorrhoea are made of natural substances and are completely safe with no harmful effects. These medicines work to root out the underlying causes of leucorrhoea and gives a completely healthy state to a person.

Keywords: Leucorrhoea, reproductive health, gynaecological problem, homoeopathy

\section{Introduction}

The complaint of vaginal discharge (leucorrhoea) is very common, particularly in India, it is associated with considerable disability, health seeking, and associated costs. This problem may occur due to unhygienic conditions, infection of genital tract or impaired immune function. Unfortunately, most women are highly embarrassed by this problem and most of them avoid discussing the problem with their medical health providers. That's why the treatment of the leucorrhoea is a bit challenging to us.

\section{Definition}

The term leucorrhoea should be restricted to those conditions when the normal vaginal secretion is increased in amount. In such patients, there will be no excess of leucocytes present when the discharge is examined under the microscope, and the discharge is macroscopically and microscopically nonpurulent ${ }^{[1]}$.

\section{OR}

Leucorrhoea is strictly defined as an excessive normal vaginal discharge. The symptoms of excessive is a subjective one with individual variation, while to declare it to be normal and not an infective one, requires and laboratory investigations. The term leucorrhoea should fulfil the following criteria:

- The excess secretion is evident from persistent vulval moisteness or staining of the undergarments (brownish yellow on drying) or need to wear a vulval pad.

- It is non-purulent and non-offensive.

- It is non-irritant and neve causes pruritis ${ }^{[2]}$.

Purulent discharges due to specific infections such as gonorrhoea, trichomoniasis and moniliasis, ulcerated growths of the cervix and the vagina. and discharges caused by urinary fistulae are of a different type and should be excluded from the term 'leucorrhoea'. Some clinicians use the term to describe any white or yellowish-white discharge from the vagina. An increase in the normal vaginal secretion develops physiologically at puberty, during pregnancy, at ovulation and, in some women, during the premenstrual phase of the menstrual cycle. During pregnancy, the normal discharge is increased in amount because of the vascularity of the female genital tract. During the latter part of the menstrual cycle, the hypertrophied premenstrual glands of the endometrium secrete mucus which is discharged through the cervix into the vagina. The leucorrhoea of puberty is probably caused by the increased vascularity of the uterus, cervix and vagina at that time. It is of temporary duration and needs no treatment. This secretion contains proteins, polysaccharides, amino acids, enzymes and immunoglobulins ${ }^{[1]}$. 


\section{Aetiology}

The excessive secretion is due to

Physiological excess: Normal secretion is expected to increasein conditions when the oestrogen levels become high. It increases naturally during puberty, during menstrual cycle(around ovulation, premenstrual pelvic congestion etc.,), during pregnancy and during sexual excitement ${ }^{[2]}$.

Cervical cause: Mucous discharge from the endocervical glands increases in such conditions as chronic cervicitis, cervical erosion, mucous polypi and ectropion. When the mucous secretion of the cervix is produced in excess, it undergoes little change in the vagina and appears as mucoid discharge at the vulva ${ }^{[1]}$.

Vaginal causes: increased vaginal transudation occurs in conditions associated with increased pelvic congestion. The conditions are- uterine prolapse, acquired retroverted uterus, chronic pelvic inflammation, pill user and vaginal adenosis [2].

\section{Some other factors may be responsible to develop white discharge}

- Hormonal imbalance especially oestrogen imbalance

- Spread of infection from urinary tract

- Early/young age pregnancy

- Ill health and poor hygiene

- Chronic sickness

- Diabetes and anaemia or any systemic disease which lowers immunity.

- Mental anxiety and sexual frustration.

\section{Symptoms}

Main symptoms are excessive vaginal discharge, pain in the thighs and calf muscles and burning micturition. The vaginal discharge color may be whitish, yellowish, reddish and blackish. The discharge may be accompanied with foul smelling and itchy sensation. Some other symptoms may be-

- Pain and heaviness in lower abdomen.

- Constipation

- Anemia

- Local soreness

- Lumbago

- Malaise

- Breathlessness

- Headache and giddiness

- Indigestion

- Anorexia

- General weakness

- Polyuria

- Pruritus

\section{Investigations and Diagnosis ${ }^{[3]}$ :}

General examination may reveal ill health.

Vulval inspection reveals: white or creamy discharge, no evidence of pruritus.

Inspection of the vagina and cervix by speculum and microscopy of a fresh wet specimen of the discharge will usually provide enough information for the diagnosis to be made and for treatment to be instituted. It reveals: either a negative pathology, associated pelvic lesion mentioned earlier causing cervical or vaginal leucorrhoea.

To exclude the infective nature, the discharge is subjected to microscopic examination for detection of pus cells; if pus cells are not detected-true leucorrhoea, if pus cells detectedidentify the organism from the discharge, these examinations include:

- Hanging drop preparation-Reveals presence of motile trichomonas organisms

- Gram stain-This may reveal presence of gram negative intracellular and extracellular diplococci suggestive of gonococci, Clue cells suggestive of bacterial vaginosis.

- Culture-

- Chocolate Agar-Gonococci

- Sabouraud's medium or Nickerson's mediumCandida $\mathrm{n}$

- Special enriched medium-Trichomonas.

- Trichomonas infection.

Preventive measures for leucorrhoea ${ }^{[4,5,6]}$.

Fresh curds must become an integral part of the diet because it not only helps in easy digestion of food but also contain lactic acid, which can reduce the discharge.

Clean the innerwear and yourself with a good quality detergent which has bactericidal and fungicidal properties.

Immediately change the clothes including undergarments, if clothes get wet in the rain or due to any other reason.

Inner wear made of Nylon material should be avoided in summer because as it may retain sweat in the genital area. Cotton is best choice for undergarments. $\varpi$ Do not unnecessarily use any cosmetics like powders or perfumes in the genital area. They should be strictly avoided.

Go on a walk or jog early morning to make the body stressfree and also to increase its resistance to diseases

Pill users should stop the pill temporarily if the symptoms is very mush annoying.

Before going physical with your partner make sure he is free from all kinds of infections and habituate yourself to wash your organ clean after meeting thus keep away many diseases.

Cleanliness of reproductive organs is very important. Wash the genitals carefully during every bath and do not let moisture retain in the genitals area after the bath. Let the water flow on anus and vulva in plenty as to wash them clean. Also wash vagina clean after urinating.

Self-medication should be avoided because some women are allergic to certain kinds of medicines and use of such medicine may cause further infections and will complicate the issue.

Drink plenty of water to flush out the toxic substances from the body.

All sugary foods such as pastries, sweets, custards, icecreams and puddings must be avoided if there is profuse discharge.

Mushrooms must be avoided in diet as they are fungi themselves.

Hot and spicy foods should be reduced to the least in diet. Avoid the intake of alcohol.

Stress buster exercises and morning walk should be made routine; because when body is stress free, immunity will receive a boost against illnesses.

Homoeopathic approach towards leucorrhoea ${ }^{[7]}$ :

Any discharge that is not blood is called leucorrhoea It is not a disease, but the product of diseased state or condition of the system. Clinically we have 4 divisions of leucorrhoea. 


\begin{tabular}{|c|c|c|c|}
\hline Vulvular & Vaginal & Cervical & uterine \\
\hline MC in children and in aged women & During child bearing period, in young women & During child bearing period & $\begin{array}{c}\text { During child } \\
\text { bearing period }\end{array}$ \\
\hline $\begin{array}{l}\text { Sero-purulent discharge, viscid, unctuous, } \\
\text { having an odour of old cheese. }\end{array}$ & $\begin{array}{l}\text { Creamy or white, purulent, may be either } \\
\text { bland or excoriating. }\end{array}$ & \begin{tabular}{|l} 
Thick, tenacious, ropy, or stringy \\
showing the albuminous elements.
\end{tabular} & \\
\hline
\end{tabular}

By the homoeopathic point of view we find that with every case the phenomena of some chronic miasm, active or latent in the organism, slumbering by virtue of the presence of the leucorrhoeal discharge or awakened by its suppression. Dr. A C Cowperthwaite says: "The constitutional or symptomatic treatment of leucorrhoea is of the greatest importance. It must be continually borne in mind that leucorrhoea is, in itself, but a symptom, either of some constitutional dyscrasia or of some local existing causes." During miasmatic analysis we find that-

Psoric discharge is- whitish albuminous, odourless and usually bland.

Sycotic discharge is -thin, watery, greenish-yellow or dark, like dirty water and usually acrid and excoriating, producing pruritus of the parts passed over. The odour is pungent, musty like fish brine or stale fish.

A syphilitic discharge may be thin, mixed with blood or may found with any malignancy. It odours foul.

Tubercular discharge is- thick, yellowish, creamy or greenish-yellow. It often has a sweetish odour.

Homoeopathic therapeutics for leucorrhoea ${ }^{[8,9,10,11]}$. Apis mellifica- Leucorrhoea profuse, yellow or green and acrid, attended with stinging sensation in the perineum. Cutting and stinging pain in the right ovary; adapted to frivolous and jealous girls, who, though generally cautious and careful, drop things or let them fall while handling them.

Ammonium muriaticum- Adapted to despondent, suicidal and sanguine temperaments; muddy complexions, light haired women or girls at puberty. Leucorrhoea profuse yellow or white, thick, corrosive, with burning and smarting of the vulva. It often excoriates the whole perineum and inner surface of thighs. It constantly runs from vagina, scrofulous and syphilitic leucorrhoea in melancholic individuals, whose minds are constantly turned towards self destruction.

Borax - Discharge like white of an egg with sensation as if warm water were flowing down. Leucorrhoea profuse, albuminous, starchy. For two wee between the catamania.

Calc carbonica- In infants and before puberty. Discharge like milk and itching and burning, worse immediately before menses; too early and profuse menses. Corrosive leucorrhoea in young children.

Graphitis - discharge very thin, watery white mucus, occurring in gushes day and night, often acrid and excoriating, great weakness in small of back when walkingorsitting; before and after menses.

Hydrastis Canadensis -Leucorrhoea worse after menses; acrid and corroding, shreddy, tenacious, ropy, thick, yellow, viscid; Hanging from os in long strings. Great weakness, constipation, pruritus vulva with profuse leucorrhoea.

Kreostum: putrid leucorrhoea which causes swelling and corrodes the parts, and there is itching as well as biting externally; leucorrhoea is exhausting; it may be milky, whey-like, or yellow. It staining linen yellow and stiffening like starch, with great weakness in legs; or white, having the odour of green corn with pain in small of back. Leucorrhoea is worse between periods.

Pulsatilla- Discharge thick like cream or milky; thick yellowish and bland but sometimes corrosive and burning with itching of parts. Thick, white mucus; discharge taking place immediately before during and after menstruation.

Sepia- discharge like milk, only in daytime; with soreness of pudenda. Cheesy or curd like discharge, which is horribly offensive. Ball like sensation in vagina and uterus associated with leucorrhoea uterine troubles.

Conclusion -Leucorrhoea is another term for vaginal discharge that is usually whitish or yellowish in colour. The discharge is a mixture of cells and fluids that is continuously released through a women's vagina. It is most commonly found problem in gynaecological OPDs. Many factors are responsible for it i.e. ill health, bad hygiene, sedentary life, hormonal imbalances, menstrual irregularities, mental stress and so on. Homoeopathy works wonderfully in cases of leucorrhoea. Homeopathy treats the person individually. Homoeopathic medicines works in such manner which provide a person complete and balanced health in a rapid way and the progress of this treatment is gentle and permanent.

\section{References}

1. Bourne, Howkins, Shaw's Textbook of Gynaecology, $16^{\text {th }}$ edition, New Delhi, Reed Elsevier India P. Ltd. 2015, 382 .

2. Dutta DC. textbook of Gynaecology, revised and enlarged $5^{\text {th }}$ edition, Kolkata, new central book agency P. Ltd. 2008, 524-26

3. Barr Wallace, clinical gynaecology, Longman group limited. 1971, 55-59

4. Leucorrhoea: Causes, symptoms, diagnosis, treatment and prevention [Internet]. Updated: 2019 December 24 [cited 2020 March 30]. Available from: https://www.thehealthsite.com/diseasesconditions/disea ses-conditions-womenshealth/leucorrhoea-causessymptoms-diagnosistreatment-and-prevention-238988/

5. Bourne A, Bond LT, Mc Garrity KA. New Treatment for Leucorrhoea [Internet]. Br Med J 1937;1(3967): 116-119, 130-3-130-4., doi: 10.1136/bmj.1.3967.116 [cited 2020 March 30]. Available from: https://www.ncbi.nlm.nih.gov/pmc/articles/PMC20880 22/

6. Gardner GH. Leucorrhoea - differential diagnosis and treatment [Internet]. Q Bull Northwest Univ. Med Sch. Winter 1940;14(4):236-241. [Cited 2020 March 30]. Available from: https://www.ncbi.nlm.nih.gov/pmc/articles/PMC38023 $10 /$

7. Allen JH. The Chronic Miasms, volume $1^{\text {st }}$, reprint edition, New Delhi, Indian Books \&Periodicals Publishers. 358-64.

8. Bhanja KC. The Homoeopathic Prescriber, $6^{\text {th }}$ edition, Pressmen Service, Kolkata, 2002, 247.

9. Allen HC. Keynote and Characteristics with 
comparisons of the leading remedies of Materia Medica with Bowel Nosodes, $8^{\text {th }}$ edition, $47^{\text {th }}$ impression. New Delhi B. Jain Publishers P. 1td. 2013, 63-73.

10. Boericke W. New manual of Homoeopathic Materia medica with Repertory, $3^{\text {rd }}$ revised and augmented edition, B Jain Publishers P. Itd., New Delhi. 2018, 274-518.

11. Choudhuri NM. A Study on Materia Medica, $11^{\text {th }}$ Impression, New Delhi B. Jain Publishers P. 1td. 2014, 489-560. 